\title{
Estudo piloto da prevalência de neuropatia periférica em adultos diagnosticados com Diabetes mellitus tipo 2 na Atenção Primária em saúde de Divinópolis - Brasil
}

\author{
Pilot study on peripheral neuropathy prevalence in adults diagnosed with type 2 Diabetes mellitus \\ in Primary Health care in Divinópolis - Brazil \\ Estudio piloto de prevalencia de neuropatía periférica en adultos diagnosticados con Diabetes \\ mellitus tipo 2 en Atención Primaria de salud en Divinópolis - Brasil
}

\section{Resumo}

Introdução: O diabetes mellitus do tipo 2 (DM2) assume grande impacto na saúde coletiva pela sua importância epidemiológica. Quanto às suas complicações, estas podem ser de caráter macro e microvascular. A nível microvascular, a neuropatia periférica diabética (NPD) assume um papel de considerável relevância, sendo essa a mais comum e incapacitante. Sua prevalência tem relação estreita com a população estudada, que varia entre 13 a 47\%. Objetivo: Realizar um projeto piloto que sirva de modelo à mensuração da prevalência de NPD em uma determinada população de pacientes diabéticos, tomando-se como exemplo a cidade de Divinópolis - Brasil. Métodos: Foram selecionadas de forma aleatória 5 unidades básicas de saúde em Divinópolis e dessas obteve-se também de maneira aleatória uma amostra de 75 usuários diagnosticados com DM2. Feita a seleção, foi aplicado o Instrumento de Classificação de Neuropatia de Michigan (MNSI-BRASIL). Tal instrumento forneceu pontuações que podem sugerir a presença de NPD por meio de um questionário auto aplicado e exame físico dos pés. Resultados: Aplicado o Instrumento de Classificação de Neuropatia de Michigan (MNSI-BRASIL), encontrou-se uma prevalência de NPD de 44\%, sendo que a prevalência em cada unidade foi: Belvedere II $40 \%$, São José 56\%, Serra Verde 38, 46\%, Central 66,6\% e Niterói 33,3\%. Conclusão: Os achados demonstram pontos positivos e negativos quanto ao desenho de estudo empregado e o instrumento de avaliação utilizado. Dessa forma, o presente trabalho servirá de substrato para estudos futuros de prevalência de NPD em Adultos com DM2.

Palavras-chave: Neuropatias diabéticas; Dados do projeto piloto; Diabetes mellitus tipo 2. 


\begin{abstract}
Introduction: Type 2 diabetes mellitus (DM2) greatly impacts public health due to its epidemiological importance. As for its complications, these can be macro and microvascular. At the microvascular level, diabetic peripheral neuropathy (DPN) plays a role of considerable relevance, being this the most common and disabling. Its prevalence is closely related to the population studied, which ranges from 13 to $47 \%$. Objective: To carry out a pilot project that serves as a model for measuring the prevalence of DPN in a determined population of diabetic patients, taking as an example the city of Divinopolis - Brazil. Methods: Five primary health care units in Divinópolis were randomly selected and a sample of 75 users diagnosed with DM2 was also randomly obtained. After selection, the Michigan Neuropathy Classification Instrument (MNSI-BRASIL) was applied. This instrument provided scores that may suggest the presence of NPD through a self-administered questionnaire and physical examination of the feet. Results: After applying the Michigan Neuropathy Classification Instrument (MNSI-BRASIL) a prevalence of NPD of $44 \%$ was found, and the prevalence in each unit was: Belvedere II 40\%, São José 56\%, Serra Verde 38, 46 \%, Central 66.6\% and Niterói 33.3\%. Conclusion: The findings demonstrate positive and negative points regarding the study design used and the assessment instrument used. Thus, the present work will serve as a substrate for future studies on NPD prevalence in Adults with DM2.
\end{abstract}

Keywords: Diabetic neuropathies; Preliminary data; Diabetes mellitus type 2.

\title{
Resumen
}

Introducción: La diabetes mellitus tipo 2 (DM2) tiene un gran impacto en la salud pública por su importancia epidemiológica. En cuanto a sus complicaciones, estas pueden ser macro y microvasculares. A nivel microvascular, la neuropatía periférica diabética (DPN) tiene un papel de considerable relevancia, siendo esta la más común e invalidante. Su prevalencia está estrechamente relacionada con la población estudiada, que oscila entre el 13 y el $47 \%$. Objetivo: Realizar un proyecto piloto que sirva de modelo para medir la prevalencia de NPD en una determinada población de pacientes diabéticos, tomando como ejemplo la ciudad de Divinópolis - Brasil. Métodos: Se seleccionaron aleatoriamente cinco unidades básicas de salud de Divinópolis y también se obtuvo aleatoriamente una muestra de 75 usuarios diagnosticados de DM2. Después de la selección, se aplicó el Instrumento de Clasificación de Neuropatía de Michigan (MNSI-BRASIL). Este instrumento proporcionó puntuaciones que pueden sugerir la presencia de NPD a través de un cuestionario autoadministrado y un examen físico de los pies. Resultados: Después de aplicar el Instrumento de Clasificación de Neuropatía de Michigan (MNSI-BRASIL) se encontró una prevalencia de NPD del 44\%, y la prevalencia en cada unidad fue: Belvedere II 40\%, São José 56\%, Serra Verde 38, 46\%, Central 66,6\% y Niterói 33,3\%. Conclusión: Los resultados evidencian puntos positivos y negativos con respecto al diseño de estudio utilizado y el instrumento de evaluación utilizado. De esa forma, el presente trabajo servirá de sustrato para futuros estudios sobre la prevalencia de NPD en adultos con DM2.

Palabras clave: Neuropatías diabéticas; Datos preliminares; Diabetes mellitus tipo 2.

\section{Introdução}

O diabetes mellitus (DM) é uma doença multifatorial, caracterizada por alterações na secreção e resistência à ação da insulina, além de uma desregulação na produção hepática de glicose. Estimou-se em 2017 que 8,8\% da população mundial vivia com DM. Essa entidade pode ser dividida em alguns tipos, sendo os tipos os 1 e 2 mais prevalentes. O diabetes mellitus tipo 2 (DM2) representa cerca de 90 a $95 \%$ dos casos e trata-se de um agravo multifatorial relacionado à interação de fatores genéticos e ambientais em sua etiologia (Sociedade Brasileira de Diabetes [SBD], 2020).

No Brasil, o DM2 tem assumido um papel central na saúde coletiva nas últimas décadas, frente ao rol de alterações que se sucederam a partir das transições epidemiológica, nutricional e demográfica observadas, sendo que dentre elas se destaca a emergência das Doenças Crônicas Não-Transmissíveis (DCNT), grupo no qual a DM2 se insere (Schmidt et al., 2011; Schramm et al., 2004).

Dentre as complicações decorrentes do diabetes, destacam-se os distúrbios macrovasculares que resultam em aumento da morbimortalidade cardiovascular. Além disso, os acometimentos microvasculares como a retinopatia, a doença renal diabética e a neuropatia periférica são importantes comorbidades associadas ao DM2 (Vilar et al,, 2016).

Dentro das principais complicações supracitadas, atribui-se grande destaque à neuropatia periférica diabética (NPD), uma vez que essa se apresenta como uma das complicações mais comuns e incapacitantes do DM2 (SBD, 2020). Tal complicação envolve um grupo heterogêneo de manifestações clínicas que afetam o sistema nervoso periférico em nível somático e autonômico (Nascimento et al., 2016). 
Sabe-se que os nervos periféricos possuem vascularização arterial por dois sistemas microvasculares distintos: um deles envolvendo o endoneuro destes nervos (intrínseco), enquanto o outro o epineuro (extrínseco). Nesse sentido, a fisiopatologia da NPD baseia-se no comprometimento de ambas as formas de suprimento sanguíneo pelo descontrole glicêmico crônico, levando a múltiplos episódios isquêmicos que culminam com morte celular gradativa de pequenas populações neuronais que compõem estes nervos, até um ponto em que essa disfunção se manifeste clinicamente e de forma irreversível (Oliveira, 2015).

Em relação à vascularização endoneural, principal sistema acometido, a hiperglicemia crônica leva a uma falha na autorregulação vasomotora deste sistema, que impede uma boa regulação local do fluxo sanguíneo em resposta às mudanças físico-químicas teciduais que acontecem a todo momento (como resultantes de alterações na taxa metabólica destes nervos, da saturação sanguínea e de outros elementos ligados à dinâmica do metabolismo tissular). O resultado dessa falta de autorregulação é uma maior vulnerabilidade dos neurônios a múltiplas isquemias. Em adição ao acometimento dos microvasos que irrigam diretamente o neurônio, a microangiopatia pode lesar as células de Schwann que revestem estes neurônios, culminando na degeneração mielínica e dano axônico, sendo este um provável mecanismo lesivo adicional (Oliveira, 2015).

A NPD é predominantemente sensitiva e com distribuição simétrica, afetando principalmente os segmentos distais dos nervos, sendo esse o principal mecanismo de ocorrência do pé diabético. As principais manifestações clínicas de comprometimento somático são dormência, queimação, "formigamento" e perda da sensibilidade, além da possibilidade de ocorrência de manifestações autonômicas concomitantes. Em alguns casos, porém, pode-se não haver sintomas ou, raramente, apenas manifestações clínicas decorrentes de disfunção motora (Nascimento et al., 2016).

Alterações nas fibras nervosas responsáveis pela motricidade surgem nos estágios mais avançados, expressas como fraqueza e diminuição da massa muscular dos pés e pernas, além de deformidades típicas como dedos em garra ou em martelo, proeminência de cabeças de metatarsos e arco plantar acentuado, resultando em pressões plantares aumentadas. Por outro lado, os efeitos da disautonomia periférica incluem a anidrose, algo que favorece as calosidades e, por conseguinte, a ulceração (SBD, 2020).

O diagnóstico das formas mais frequentes de NPD baseia-se na caracterização do quadro clínico com os sinais e sintomas mais típicos e na realização de testes neurológicos. A avaliação também pode ser feita através de ferramentas de screening, a partir de dados demográficos, clínicos e sobre o estilo de vida do diabético, pela avaliação clínica dos pés, por testes neuropáticos e pelo índice tornozelo-braquial (ITB) (Brinati et al., 2017).

O diagnóstico da NPD é essencialmente clínico, e sua prevalência é diretamente influenciada pela população estudada e pelo critério diagnóstico utilizado, o que se reflete nos indicadores de prevalência em saúde. Assim sendo, encontra-se uma prevalência em estudos populacionais e pacientes ambulatoriais que varia na faixa de $13 \%$ a $47 \%$. A incidência da neuropatia diabética é de aproximadamente $2 \%$ ao ano em pacientes recentemente diagnosticados com DM, sendo que estes valores aumentam para $6 \%$ a $10 \%$ ao ano em pacientes com mais tempo de doença, de modo que a polineuropatia diabética distal, sua forma mais comum, chega a afetar metade dos pacientes após cerca de 20 anos de doença (Moreira et al., 2005).

Para delineamento de estudos consistentes que forneçam indicadores de real relevância, faz-se necessário a realização do presente estudo piloto, uma modalidade de trabalho que tem como objetivo avaliar a performance, potencial e a viabilidade de um possível desenho de estudo subsequente (Bril \& Perkins, 2002).

Conhecer os indicadores da NPD é fundamento básico para planejar ações de saúde preventiva. Além disso, a identificação precoce do processo da NPD reforça no paciente diabético uma necessidade maior de controle dos índices glicêmicos, que associada a medidas de higiene e cuidado com os pés, possibilitam a prevenção de complicações, como por exemplo, as amputações (Ochoa-Vigo et al., 2006; De Mettelinge et al., 2013). 
A obtenção de estatísticas precisas sobre a dimensão deste fenômeno no município de Divinópolis será uma ferramenta para o diagnóstico epidemiológico em saúde local e servirá como um substrato para adoção de políticas públicas eficazes que busquem a reversão de um provável quadro de subdiagnóstico.

O objetivo geral do presente estudo foi realizar um projeto piloto que sirva de modelo à mensuração da prevalência de NPD em uma determinada população de pacientes diabéticos, tomando-se como exemplo os usuários da atenção primária do município de Divinópolis - Brasil. Os objetivos específicos incluíram a produção de substrato para a elaboração de estudos futuros que fornecerão indicadores que poderão auxiliar no sistema de saúde, avaliação da viabilidade do desenho de estudo utilizado, seleção de pontos positivos e negativos quanto o instrumento de avaliação empregado e fornecimento de um relatório às unidades básicas de saúde e/ou ESF acerca dos participantes recém diagnosticados a fim de que fossem devidamente acompanhados ou encaminhados para os serviços de referência.

\section{Metodologia}

\subsection{Desenho de estudo e cálculo amostral}

Trata-se de um estudo piloto do tipo seccional, que se caracteriza pela estratégia de observação transversal, esquema de seleção incompleto e unidade de observação individual, com predomínio de uma análise de caráter descritiva (Medronho et al., 2008). O método de amostragem aleatória definido para o estudo em descrição é do tipo estratificado, que consiste em dividir a população em grupos relativamente homogêneos e mutuamente exclusivos, chamados estratos e selecionar amostras aleatórias simples e independentes em cada estrato (Reis et al., 2006).

O processo de amostragem foi aplicado sobre os dados fornecidos pela Secretaria Municipal de Saúde do município de Divinópolis (SEMUSA). Sabe-se que a população total de Divinópolis em 2018, ano de início do delineamento do estudo, era de 235.977 habitantes, na qual 191.277 estavam cadastrados no Sistema de Informação em Saúde (SIS), o qual contém registro de informação em saúde dos usuários do Sistema Único de Saúde (SUS) em Divinópolis.

No SIS, em 2018, haviam 5103 usuários do SUS em Divinópolis cadastrados como diabéticos segundo a categoria de Doença ou Condição Referida, correspondendo à população em estudo. A este montante definiu-se como estratos as Unidades Básicas de Saúde (UBS) ou Estratégias de Saúde da Família (ESF), após o levantamento da quantidade de pacientes que estão na abrangência de cada uma delas. Em seguida, avaliou-se o número de pessoas a ser selecionado em cada UBS ou ESF a partir do tamanho do respectivo estrato, de modo proporcional ao tamanho da abrangência de cada um deles. Por fim, empregou-se um método de seleção aleatória simples para definir quais destes usuários serão escolhidos para compor a amostra final.

Os pacientes diabéticos, com registro no SIS, estavam distribuídos em 43 ESFs ou UBSs. A distribuição relativa era marcadamente heterogênea, já que se observou desde 9 até 328 participantes por unidade. O número de participantes selecionados por unidade variou na mesma proporção, sendo os limites inferior e superior 2 e 29 participantes, respectivamente.

Justifica-se a escolha desse tipo de amostragem em razão do pressuposto de que a estratificação de uma população é válida quando é possível identificar subpopulações que variam muito entre si no que diz respeito à variável em estudo, mas que apresentam poucas diferenças dentro de cada uma delas. Isso é ainda mais importante se a variável a ser estratificada se encontrar correlacionada com outras variáveis como, por exemplo, idade, sexo, rendimento, status, área geográfica, entre outras (Reis et al., 2006).

Em outras palavras, visto que a NPD está diretamente relacionada às condições de cuidado que são fornecidas ao usuário pelo sistema de saúde, é possível discriminar importantes diferenças entre as diversas unidades de saúde e este construto mostrase primordial para avaliar sua eficácia no que tange à terapêutica empregada para controle do DM2. Desta maneira, para 
realização do estudo piloto foram selecionadas 5 unidades básicas de saúde e dessas obteve-se também aleatoriamente, 75 pacientes adultos diagnosticados com DM2.

\subsection{Escala para Classificação de Neuropatia Periférica}

Para avaliar a presença de NPD, foi empregado o Instrumento de Classificação de Neuropatia de Michigan (MNSIBRASIL), que foi desenvolvido no Centro de Treinamento e Pesquisa da Universidade de Michigan, nos Estados Unidos e validado por Moghtaderi em 2006, apresentando sensibilidade de 79\% e especificidade 94\% (Quadros 1 e 2). Tal instrumento foi traduzido e adaptado transculturalmente para o Brasil, em 2015. Ao final da validação, obteve-se um coeficiente de correlação Intraclasse $(\mathrm{CCI})$ com alta confiabilidade do tipo interavaliadores $(\mathrm{CCI}=0,840)$ quanto do tipo teste-reteste $(\mathrm{CCI}=0,864)$, considerados excelentes (Oliveira, 2015).

Sabe-se que não existe um instrumento padrão-ouro consagrado na literatura como ferramenta universal para o diagnóstico de NPD. Portanto, a escolha deste instrumento, em detrimento dos demais de mesmo objetivo, baseou-se no fato de ser a ferramenta diagnóstica mais nova e também promissora, já que possibilita avaliação da história clínica aliada ao exame físico, com alta especificidade e confiabilidade interavaliadores, de forma prática e de eficácia comparável aos testes eletroneuromiográficos, considerados padrão-ouro para o diagnóstico, porém bem menos acessíveis.

O MNSI-BRASIL é composto por uma introdução com orientação sobre sua utilização, seguida por duas partes para aplicação. A primeira parte (A), auto administrada pelo participante, foi preparada para pontuar a história clínica e a segunda parte (B), preenchida pelo pesquisador, para a avaliação física (Oliveira, 2015).

Quadro 1 - Parte A do MNSI-BRASIL: História clínica.

\begin{tabular}{|l|l|}
\hline \multicolumn{1}{|c|}{$\begin{array}{l}\text { Por favor, tire alguns minutos para responder as seguintes perguntas sobre a sensibilidade de suas pernas e } \\
\text { pés. Marque sim ou não baseado em como você usualmente se sente. Obrigado. }\end{array}$} & $\square$ Sim \\
\hline 1. Você sente que suas pernas e/ou pés estão dormentes? & $\square$ Sim \\
\hline 2. Você já sentiu alguma dor em queimação em suas pernas e/ou pés? & $\square$ Não \\
\hline 3. Os seus pés estão muito sensíveis ao toque? & $\square$ Sim \\
\hline 4. Você tem câimbras nos músculos de suas pernas ou pés? & $\square$ Sim \\
\hline 5. Você já teve sensações de formigamento em suas pernas e/ou pés? \\
\hline 6. Suas pernas e/ou pés doem quando o cobertor toca sua pele? & $\square$ Sim \\
\hline 7. Quando você entra no banheiro ou no chuveiro, você é capaz de dizer se a água está quente ou fria? \\
\hline 8. Você já teve uma ferida aberta em seu pé? & $\square$ Sim \\
\hline 9. Seu médico já te disse que você tem neuropatia diabética? & $\square$ Não \\
\hline 10. Você se sente fraco a maior parte do tempo? & $\square$ Sim \\
\hline 11. Seus sintomas pioram durante a noite? & $\square$ Sim \\
\hline 12. Suas pernas doem quando você caminha? & $\square$ Não \\
\hline 13. Você é capaz de sentir seus pés enquanto caminha? & $\square$ Não \\
\hline 14. A pele do seu pé fica seca que chega a rachar-se? & $\square$ Sim \\
\hline 15. Você já fez uma amputação? & $\square$ Sim \\
\hline Total: & $\square$ Não \\
\hline
\end{tabular}

Fonte: Adaptado de Oliveira (2016). 
Quadro 2 - Parte B do MNSI-BRASIL: Avaliação física.

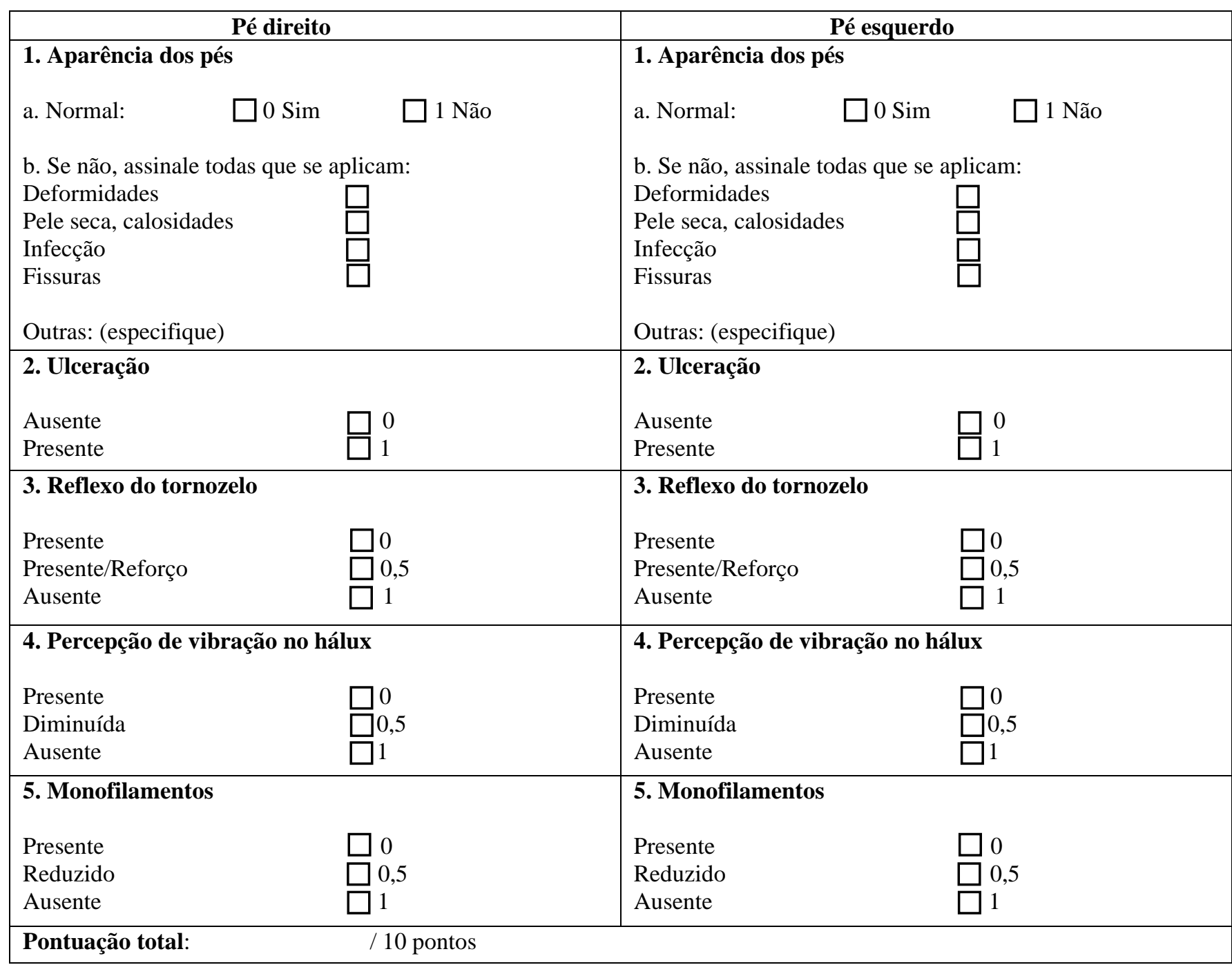

Fonte: Adaptado de Oliveira (2016).

Com relação à parte A, são analisados 15 itens, nos quais o participante poderá marcar "sim" ou "não". Respostas "sim" nos itens 1, 2, 3, 5, 6, 8, 9, 11, 12, 14 e 15 são contabilizadas com um ponto cada. Uma resposta "não" nos itens 7 e 13 contam um ponto. $\mathrm{O}$ item 4 é uma medida de déficit circulatório e o item 10 é uma medida de astenia geral e não são incluídas na pontuação, mas foram desenvolvidas para servir de substratos a outras avaliações clínicas posteriores. As descrições das pontuações não estão presentes no questionário que o paciente responderá. Após o preenchimento do questionário, o pesquisador deve registrar a pontuação obtida na parte A (Oliveira, 2015).

Quanto à parte $\mathrm{B}$, todos os participantes da pesquisa devem estar com pé aquecido $\left(>30^{\circ} \mathrm{C}\right)$ (Oliveira, 2015). A temperatura foi aferida por meio de um termômetro digital com infravermelho. Também foi solicitado aos participantes que no dia da participação, deveriam apresentar-se na unidade com sapatos fechados. Caso essa condição não fosse atendida e a temperatura inadequada, foi solicitado o retorno em uma data futura.

Todos os procedimentos da parte B devem ser repetidos em ambos os pés, sendo que o pé direito será avaliado primeiramente. São analisados 5 itens: 1 - Aparência dos pés; 2 - Ulceração; 3 - Reflexos do tornozelo; 4 - Percepção de vibração do hálux e 5 - Monofilamentos (Oliveira, 2015).

O primeiro item (Aparência dos pés), corresponde a inspeção dos pés e classificação quanto à normalidade (subitem a). Se considerado normal, atribuiu-se a pontuação 0 e prossegue-se para o item 2. Se considerado não normal, pontua-se 1 e deve 
assinalar as anormalidades que se aplicam (subitem b). Nesse sentido foi possível assinalar a ocorrência de deformidades que, por definição, foram consideradas: pés planos, dedos em martelo, dedos sobrepostos, hálux valgo, subluxação articular, cabeças metatarsais proeminentes, convexidade medial (pé de Charcot) e amputação. Além disso, pode ser encontrada pele seca, calosidades, infecção, fissuras ou outras que podem ser especificadas em campo reservado (Oliveira, 2015).

O segundo item (Ulceração), se presente, recebe pontuação 1 e se ausente, pontuação 0 . O terceiro item (Reflexos do tornozelo) foi testado utilizando um martelo neurológico. Os reflexos de estiramento muscular do tornozelo foram evocados na posição sentada, com o pé suspenso e o participante relaxado. No momento do reflexo, o pé foi passivamente posicionado e levemente dorsofletido para se obter o alongamento ótimo do músculo. O tendão de Aquiles foi percutido diretamente e, se o reflexo fosse obtido, foi graduado como presente e recebeu a pontuação 0. Se o reflexo estivesse ausente, o participante era orientado a realizar a manobra de Jendrassik, que consiste em colocar os dedos das mãos de modo entrelaçado e tracionados no sentido oposto, uns contra os outros, de modo a "distrair" o participante. Reflexos evocados somente durante realização dessa manobra foram definidos como "presente com reforç̧" e receberam a pontuação 0,5 . Se o reflexo não estivesse presente mesmo com a execução da manobra, foi considerado ausente (Oliveira, 2015).

O quarto item (Percepção de vibração no hálux) avalia a sensibilidade vibratória utilizando-se um diapasão de $128 \mathrm{~Hz}$ colocado sobre o dorso do hálux, livre de apoio, na proeminência óssea da articulação interfalangeana. Os participantes estavam com os olhos fechados e foram solicitados a indicar quando sentissem o término da vibração proveniente do diapasão. Para testar se a intensidade de vibração aplicada era ideal, o examinador sentia a vibração da mão que segurava o diapasão, na região distal de seu dedo indicador, cerca de 5 segundos depois que um sujeito normal podia senti-la em seu hálux. Assim, se o examinador sentisse a vibração depois de 10 ou mais segundos em seu dedo indicador concluía-se que a vibração era insuficiente para o teste. A vibração foi pontuada como: 1) presente, se depois que o participante relatasse o término da vibração o examinador ainda a sentisse em seu dedo por um período < 10 segundos, situação em que recebeu pontuação $0 ; 2$ ) diminuída, se sentisse por período $\geq 10$ segundos e recebeu-se a pontuação 0,5 ; ou 3) ausente, se o participante não detectasse a vibração (Oliveira, 2015).

O quinto item (Monofilamentos), avalia a sensibilidade cutânea e, para isso, o pé do participante teve que estar apoiado. Foram utilizados para essa etapa, Monofilamentos Semmes-Weinsten de 10 gramas (estesiômetro), que foram aplicados no dorso do hálux, $2^{\circ}$ e $5^{\circ}$ metatarsos, em três pontos do antepé, em um ponto do mediopé e calcâneo de forma breve e perpendicularmente ( $<1$ segundo) e os participantes, ainda com os olhos fechados, foram solicitados a responder positivamente caso sentissem o filamento. O conjunto de oito respostas corretas em 10 aplicações foi considerado normal. O conjunto de uma até sete respostas corretas indicou sensibilidade reduzida e nenhuma resposta correta foi compreendida como ausência de sensibilidade (Oliveira, 2015).

Após a aplicação das partes A e B, somam-se as pontuações de cada parte para obter-se a pontuação total. Tal valor, quando maior que 8, sugere neuropatia periférica simétrica (Oliveira, 2015).

\subsection{Aplicação do MNSI-BRASIL}

Para a realização da avaliação foram necessárias uma sala, uma mesa e duas cadeiras para a aplicação do Termo do Consentimento Livre e Esclarecido (TCLE) e da parte auto aplicada, além de uma maca para a aplicação da parte B do MNSIBRASIL. Foi necessário um estesiômetro para teste de sensibilidade tátil superficial, martelo neurológico para teste de reflexos e um diapasão de $128 \mathrm{~Hz}$ para teste de sensibilidade vibratória. Foi também fundamental a impressão de uma cópia do MNSIBRASIL e duas vias do TCLE.

Com relação ao espaço físico para a realização da pesquisa, primeiramente foi realizada uma reunião com os responsáveis pelas unidades de saúde selecionadas. Dessa forma, foram expostas e detalhadas todas as etapas e objetivos do projeto de pesquisa. Após o aval destes, a equipe de pesquisadores fez um estudo do melhor local disponível para a aplicação 
dos testes. O horário foi previamente combinado com os participantes e os responsáveis pela unidade, levando-se em conta a disponibilidade de ambas as partes. Também foi informado, no momento dessa reunião com os profissionais de cada unidade, a necessidade do agendamento da consulta para os participantes que forem diagnosticados com NPD, com data marcada já no momento do diagnóstico, para a primeira ocasião em que for possível nos dias subsequentes àquele dia.

Os participantes selecionados para a participação da pesquisa foram convidados via meios de contato disponibilizados pela UBS / ESF ou por intermédio do agente comunitário de saúde a comparecerem em dia e horários previamente determinados. Nesse convite, houve uma conversa com os pesquisadores, na qual foram explicados detalhadamente os objetivos, além dos possíveis riscos e benefícios quanto à participação no projeto. Também foi informado que o participante poderia deixar de participar do estudo a qualquer momento, sem nenhum ônus. O eventual gasto com transporte, para o local de realização da pesquisa, se necessário, foi custeado pelos pesquisadores. Além disso, foi informado a necessidade do agendamento de uma consulta com o profissional médico de sua unidade, caso o diagnóstico de NPD fosse detectado.

\subsection{Análise dos riscos e benefícios}

Foram explicitados aos participantes desta pesquisa todos os possíveis riscos através do TCLE. Além disso, foram informados quanto à liberdade de participar ou não, bem como da possibilidade de desistência no decorrer de sua participação sem nenhum prejuízo. Alguns possíveis riscos incluem cansaço ou aborrecimento ao responder questionários; constrangimento ou ocorrência de resultados falsos positivos após a aplicação do MNSI-BRASIL gerando ansiedade no participante. Porém tratase de algo esperado, visto que, como todo teste, pode haver incertezas diagnósticas.

Visando evitar ao máximo os riscos citados, é importante enfatizar que certas precauções foram tomadas durante as entrevistas e exames clínicos. Para tal, os entrevistadores passaram por uma capacitação prévia. Isso tornou-lhes mais hábeis para diminuir o tempo de entrevista. Além disso, foram evitadas perguntas desnecessárias ou constrangedoras, estando atentos aos sinais verbais e não verbais de desconforto e não estabeleceu-se juízo de valor em nenhuma hipótese. Em adição, os participantes foram acolhidos em ambiente limpo e tranquilo e foram também avisados quanto à possibilidade de não responder ou parar a entrevista e/ou exame a qualquer momento que se sentissem desconfortáveis. Se porventura algum desses danos ocorreu, a equipe de pesquisa certificou-se que o participante recebeu acompanhamento integral.

Os benefícios advindos da participação no estudo consistiam no oferecimento do diagnóstico da população afetada por essa complicação, o que possibilitou também um melhor conhecimento a respeito da realidade dos habitantes e consequentemente dos indicadores de saúde. Além disso, a nível individual, auxiliou na identificação de pessoas com NPD, permitindo orientações adequadas que visavam retardar a evolução de tal comorbidade, contribuindo para evitar, no futuro, as repercussões mais graves como, por exemplo, amputação e perda da sensibilidade dos membros do corpo.

\subsection{Capacitação}

Os pesquisadores envolvidos no estudo foram previamente capacitados pelo orientador Mauro Ézio Eustáquio Pires, médico especialista em ortopedia, com o intuito de otimizar suas habilidades semiotécnicas para a aplicação devida MNSIBRASIL. A capacitação foi realizada em três encontros totalizando 15 horas, nas quais foram realizadas as práticas: exame com estesiômetro, avaliação do reflexo Aquileu, teste com diapasão e reconhecimento dos achados da NPD manifestos em inspeção clínica dos pés.

Ao final do treinamento, os pesquisadores foram submetidos a um pré-teste, que consistiu na aplicação do MNSIBRASIL entre eles, com o propósito de aperfeiçoar sua velocidade de aplicação e a precisão das perguntas feitas, bem como suscitar e questionar possíveis dúvidas que surgiriam no trabalho em campo. Pretendeu-se, dessa forma, reduzir a variabilidade 
intra e interobservador, além de minimizar possíveis efeitos negativos impostos aos participantes pela aplicação da escala, bem como a redução dos riscos previamente citados e a potencialização dos benefícios para o participante.

\subsection{Critérios de inclusão e exclusão}

Os critérios de inclusão para compor a amostra foram pacientes diagnosticados com DM2 que possuíam registro no SIS, com idade igual ou superior a 18 anos. Os critérios de exclusão englobavam a presença de amputação dos membros inferiores ao nível maleolar ou superior, pois prejudica a avaliação física do pé e a pontuação inferior a 19 pontos no Mini Exame do Estado Mental, que era realizado momentos antes da aplicação do MNSI-BRASIL, com o objetivo de garantir que o participante era capaz de compreender adequadamente os testes que seriam feitos e de responder de forma fiel às perguntas do questionário. Além disso, por se tratar de um instrumento auto aplicado, foi também critério de exclusão indivíduos não alfabetizados.

Se o convite para a participação fosse aceito, eram realizadas as avaliações, que ocorriam individualmente, na sala reservada das UBS / ESF da cidade Divinópolis, com o mínimo de interferência externa, por dois avaliadores, previamente treinados. Inicialmente, os participantes respondiam à primeira parte do MNSI-BRASIL e, em seguida, os avaliadores aplicavam a segunda parte, correspondente ao exame clínico.

\subsection{Conduta diante dos participantes com NPD e outras complicações}

Os participantes que foram devidamente diagnosticados com NPD segundo o MNSI- BRASIL receberam um Relatório de Cuidados informando-lhes sobre sua condição e os cuidados necessários para seu correto controle, além de ressaltar a necessidade de buscar um atendimento especializado e longitudinal através de sua UBS / ESF. Paralelamente, outro relatório foi enviado diretamente à UBS / ESF que o participante pertence. Esse segundo relatório pôde ser anexado ao seu prontuário, informando sobre o diagnóstico e reiterando a necessidade de uma abordagem multiprofissional dessa condição.

Ao mesmo tempo, os participantes diagnosticados com NPD tiveram esse achado registrado formalmente em seu prontuário, acrescido de uma nota informando a necessidade de consulta com o profissional médico de sua unidade. Para que esse fluxo dentro do sistema de saúde se iniciasse, o agendamento da consulta era feito pelos próprios pesquisadores com os responsáveis pela sua marcação, na secretaria da unidade, no mesmo dia do diagnóstico, para a primeira data possível. A data e o horário da consulta foram pactuados com o participante, além de serem registrados formalmente no Relatório de Cuidados que lhes foi entregue.

Desta forma, o plano de cuidado proposto para os participantes que foram devidamente diagnosticados com NPD incluiu, de imediato, o médico assistente, ao qual foi facultada a possibilidade de encaminhar o participante, se julgasse necessário, para a atenção secundária em saúde ou a outros serviços especializados. Portanto, todo participante portador de NPD teve garantia de atendimento pelo sistema de saúde em que estava inserido.

\subsection{Análise dos dados}

As informações obtidas foram transcritas e armazenadas em um banco de dados, em planilhas do Microsoft Office Excel 2016, isentos de identificação nominal, em posse exclusiva dos participantes da pesquisa, com garantia de sigilo e confidencialidade. Cada voluntário recebeu um código no momento da aplicação do MNSI-BRASIL que foi utilizado durante a análise de dados.

Por meio de estatística descritiva, os dados obtidos foram analisados por distribuição de frequências. Desse modo, os resultados foram confrontados com outros achados na literatura e abordados em detalhes na discussão. Com o decorrer do estudo foi possível, para os pesquisadores envolvidos, levantaram aspectos facilitadores e dificultadores dos métodos empregados. 


\section{Resultados}

Procedeu-se com o sorteio dentre todas as unidades de saúde de Divinópolis e as cinco selecionadas foram as seguintes, em sua respectiva ordem de sorteio: Belvedere II, São José, Serra Verde, Central e Niterói. A coleta de dados respeitou a ordem do sorteio e obteve-se ao final de todas as coletas um total de 75 participantes.

As distribuições do número de casos em cada unidade, separadamente, bem como a prevalência observada em cada UBS/ESF, estão descritas no Quadro 3. Após a aplicação do MNSI-BRASIL nas 5 unidades citadas, obteve-se um resultado de $44 \%$ para prevalência de NPD.

Quadro 3 - Diagnóstico de NPD.

\begin{tabular}{|l|c|c|c|}
\hline \multicolumn{1}{|c|}{ UBS/ESF } & $\begin{array}{c}\text { Número de } \\
\text { participantes }\end{array}$ & $\begin{array}{c}\text { Participantes } \\
\text { diagnosticados com } \\
\text { NPD }\end{array}$ & $\begin{array}{c}\text { Porcentagem de } \\
\text { diagnóstico de NPD }\end{array}$ \\
\hline Belvedere II & 10 & 4 & $40,00 \%$ \\
\hline São José & 25 & 14 & $56,00 \%$ \\
\hline Serra Verde & 13 & 5 & $38,46 \%$ \\
\hline Central & 3 & 2 & $66,6 \%$ \\
\hline Niterói & 24 & 8 & $33,33 \%$ \\
\hline Total & 75 & 33 & $44,00 \%$ \\
\hline
\end{tabular}

Fonte: Autoria própria.

\section{Discussão}

\subsection{Perspectiva do estudo piloto}

A casuística original do presente trabalho pretendia estabelecer a prevalência de NPD em pacientes diagnosticados com DM2 no município de Divinópolis - Brasil. Para isso o MNSI-BRASIL seria aplicado em todas as UBS/ESF do referido município. Porém ainda durante a fase inicial da coleta de dados, iniciou-se o período de pandemia da COVID-19.

Nesse sentido, os pesquisadores, juntamente com o professor orientador e a instituição a qual são vinculados, optaram por suspender por tempo indeterminado a coleta de dados. Tal decisão foi baseada nos dados disponíveis que ressaltavam o aumento da incidência de complicações relacionadas ao COVID-19 em pacientes com DM2. Além disso, a maior parte dos participantes da pesquisa eram idosos, perfazendo, dessa forma, dois fatores de risco concomitantes (Li et al., 2021). Portanto, diante desse contexto, por se tratar de uma doença altamente contagiosa, para resguardar a saúde dos pesquisadores e sobretudo dos participantes da pesquisa, optou-se por paralisar a pesquisa na fase de coletas.

Diante das incertezas causadas pela pandemia do COVID-19, optou-se por encerrar a coleta de dados, após a realização em cinco UBS/ESF. Nesse contexto, houve consenso entre a equipe de pesquisa em mudar o foco da pesquisa, de forma que fosse realizada a análise dos diversos fatores relacionados à aplicação do MNSI-BRASIL. Assim, tendo em vista que se procedeu a aplicação em 75 participantes, já pôde ser possível a abordagem e discussão de diversos aspectos quanto à aplicação do presente instrumento de pesquisa, em face da literatura disponível.

Dessa maneira, pretende-se com a mudança de perspectiva, a análise mais detalhada dos fatores facilitadores e dificultadores na aplicação do MNSI-BRASIL, englobando os aspectos biopsicossociais no cuidado em saúde bem como questões organizacionais do SUS. Além disso, buscou-se analisar objetivamente os aspectos práticos da aplicação da escala, ressaltando tanto as potencialidades quanto às limitações de tal método para o diagnóstico da NPD. Por fim, procedeu-se à análise dos possíveis vieses, além de comparação entre diversos instrumentos para diagnóstico de neuropatia.

Assim, o presente estudo tem como objetivo servir de estudo piloto para o desenvolvimento de estudos futuros que busquem a determinação da prevalência de NPD com elevada acurácia em determinados parâmetros populacionais. Sabe-se que 
um estudo piloto se define como um estudo preparatório, cujo objetivo seria de avaliar a performance, potencial e a viabilidade das diversas estratégias de um possível desenho de estudo subsequente e maior (Medronho et al., 2008). Sabe-se que desde que aplicado sob uma população minimamente semelhante, o estudo piloto permite subsidiar o aprimoramento da metodologia de coleta de dados, tratando-se então de uma etapa crucial para a elaboração de um projeto de pesquisa válido (Koche, 2011). Nesse sentido, pode-se concluir que esse será o contorno que moldará as discussões a seguir, com ênfase na construção de um desenho de estudo sólido que possibilite a determinação futura da NPD em diversas situações clínico-epidemiológicas, de forma mais verossímil possível.

\subsection{Fatores facilitadores e dificultadores na aplicação do MNSI-BRASIL}

O primeiro gargalo observado pelos pesquisadores foi o Sistema de Informação em Saúde (SIS), utilizado para a seleção aleatória dos participantes. Os pesquisadores, após identificar quais pacientes iriam entrar em contato, por telefone, depararamse com números inexistentes, além de alguns que não pertenciam ao respectivo participante.

Outra falha observada foi a presença de pessoas já falecidas ainda com registro do SIS, demonstrando falha na atualização dos dados, bem como a falta de integração com o Sistema de Informações sobre Mortalidade (SIM). Tal situação dificulta a geração de dados epidemiológicos consistentes e que dimensionem de forma confiável a ocorrência do DM2 e de suas complicações no município de Divinópolis. Trata-se de uma questão relevante quanto ao direcionamento de recursos e políticas públicas, pois pode haver desperdícios se há um número maior de pacientes do que o real, ou falta de recursos, se não há o cadastro suficiente dos doentes.

A presença de pessoas falecidas no SIS foi constatada apenas no momento do contato por telefone, em que familiares, ao atenderem, informavam o ocorrido. Tratava-se de situação extremamente desconfortável tanto para os familiares como para os pesquisadores, a qual, infelizmente, repetiu-se por diversas vezes. Nesse sentido, sugere-se que para estudos futuros, ao utilizar sistemas de informação como o SIS, adote-se uma abordagem de acesso aos participantes de modo padronizado e que busque contornar semelhante situação, de forma a reduzir o desconforto ocasionado em tal contexto.

Os diversos SIS desenvolvidos pelo SUS têm como objetivo ajudar a melhorar a prestação de serviços médicos e reduzir custos. São sistemas que monitoram riscos, gerenciam informações e controlam a demanda por produtividade e transferência de recursos financeiros. No entanto, por serem diferentes em relação ao banco de dados e configuração do sistema, eles apresentam dificuldades de comunicação e integração mútua. (Bittar et al., 2018).

Ao comparar a situação em outras partes do Brasil com a situação local, as observações são semelhantes. Em um estudo que analisou informações do SUS e da Secretaria de Saúde do estado de São Paulo, observou-se dificuldade de comunicação entre diferentes dados e a conversão em informações. Conclui-se, então, que há uma utilização exagerada de softwares e planilhas, sem efetiva comunicação destes instrumentos entre si (Bittar et al., 2018).

Portanto, questiona-se ainda se há o conhecimento das necessidades e demandas de saúde da população adscrita, já que isso pressupõe um conhecimento mais verossímil e atualizado desse grupo. Esse dado é fundamental, considerando-se que atenção básica deve abranger toda a população e atuar como porta de entrada do SUS, sendo responsável pela coordenação da atenção à saúde e pelo atendimento das diversas demandas em saúde. (Lima et al., 2015).

Além dos problemas já descritos, foi observado no momento do convite para participação da pesquisa, por telefone, que houveram diversas negativas alegando diversos empecilhos que não podiam ser prontamente resolvidos. Foi o caso do deslocamento para a unidade de saúde, uma vez que diversos indivíduos possuíam algum tipo de restrição de movimento, bem como falta de meios de locomoção facilmente acessíveis, associados a localização distante da UBS/ESF. Nesse contexto, é questionável se as pessoas com DM e outras doenças crônicas estão de fato sendo acolhidos pela atenção primária e recebendo um acompanhamento longitudinal, haja vista as limitações observadas pela presente casuística. 
De fato, as dificuldades observadas pelos pesquisadores, referentes ao acesso à unidade de saúde, apenas refletem uma situação cronicamente observada na atenção primária, no Brasil. O excesso de usuários vinculados à equipe da ESF, o subdimensionamento e subfinanciamento da atenção primária, número reduzido de médicos de Família e Comunidade, com pouca interiorização e fixação, além de elevada burocratização, são pontos chaves pelos quais esse problema perpassa (Tesser et al., 2018).

Para melhorar o acesso, é necessário aumentar o investimento federal neste nível de atenção, de forma a priorizá-lo e ampliá-lo. Além disso, é necessário promover a formação em medicina de Família e Comunidade, valorizar as clínicas de enfermagem, diversificar as formas de comunicação com os usuários, explorar a gestão conjunta das equipes e flexibilizar a agenda dos profissionais. Tudo isso deve ser associado com a redução do número de usuários vinculados a uma única equipe (Tesser et al., 2018).

Por outro lado, apesar dos empecilhos observados pelos pesquisadores, ressalta-se que a aplicação do MNSI-BRASIL mostrou-se como uma ferramenta relevante para acessar parte do público coberto pela atenção básica, considerando a elevada prevalência de DM2 e de suas complicações na população. Foi um instrumento que motivou diversos indivíduos que há tempo considerável não compareciam à UBS, a novamente entrarem em contato com esse aparato de saúde.

\subsection{Aspectos práticos da aplicação do MNSI-BRASIL}

Nos dias de coleta, observou-se abstenção relevante, uma vez que grande número de pessoas que aceitaram o convite para participação, por telefone, não compareceu à UBS/ESF na data e horário previamente marcados. Dessa forma, pode-se questionar a limitação da comunicação por telefone para transmitir com clareza os objetivos do projeto e os benefícios oriundos de sua participação. Para trabalhos futuros, sugere-se uma padronização através de uma abordagem sistemática, com linguagem simples para fácil apresentação dos objetivos do trabalho e dos benefícios da participação, bem como os riscos inerentes, a fim de reduzir a abstenção de participantes.

Quanto ao processo de aplicação, um instrumento auto aplicado possui diversas limitações, sendo a principal delas a necessidade de leitura e compreensão das perguntas propostas. Vários potenciais participantes foram excluídos da pesquisa por declararem-se analfabetos. Trata-se de um aspecto relevante em estudos envolvendo a população brasileira, pois segundo dados da Pesquisa Nacional por Amostra por Domicílios Contínua (PNAD), do Instituto Brasileiro de Geografia e Estatística (IBGE), no Brasil em 2019, a taxa de analfabetismo estava em 6,6\%, correspondendo a cerca de 11 milhões de pessoas (Instituto Brasileiro de Geografia e Estatística [IBGE], 2019).

Quanto aos dados de Minas Gerais, a PNAD, em 2017, demonstrava 6,0 \%, de analfabetismo da população de 15 anos ou mais. Entre as pessoas com 60 anos ou mais, 19,2\% eram analfabetos (Fundação João Pinheiro [FJP], 2018). Tal número mostra-se relevante ao se pensar em instrumentos auto aplicados para rastreio de complicações do DM2, visto que é uma doença mais presente nessa faixa etária, bem como suas complicações. Dessa forma, a parte auto aplicada do MNSI-BRASIL pode se tornar um obstáculo para estimativas mais próximas da realidade quanto à prevalência da NPD e de outras complicações do DM, por haver a possibilidade da perda de um número considerável de indivíduos. Portanto, ao analisar a realidade nacional, instrumentos que não sejam auto aplicados podem mostrar-se mais adequados.

No entanto, é necessário enfatizar que os pontos abordados na primeira parte MNSI-BRASIL se mostram importantes devido à abordagem de vários dos sintomas vivenciados pelos pacientes com neuropatia diabética (Nascimento et al., 2016). A busca ativa dessas mesmas queixas por parte do profissional responsável pode ser uma alternativa para vencer os entraves relativos à limitação de alfabetização dos participantes, sem, contudo, ignorar informações clínicas tão relevantes para o diagnóstico de NPD. 
Por outro lado, a segunda parte do MNSI-BRASIL, que consiste na aplicação do exame físico, mostrou-se de fácil aplicação. Após curto período de capacitação, os pesquisadores realizaram os testes de forma rápida e facilmente reprodutível. São testes práticos, com instrumental relativamente simples, que demonstram a presença ou ausência dos tipos de sensibilidade que podem ser perdidos com a neuropatia diabética (Nascimento et al., 2016).

Um ponto positivo da realização do projeto, em si, foi o acesso a diversos pacientes que relatavam que há muito tempo não compareciam à unidade de saúde. Dessa forma, foi uma oportunidade para prestar esclarecimentos quanto aos cuidados básicos em relação ao DM2, bem como a recomendação de agendamento de consulta médica na unidade e acompanhamento ambulatorial. Para todos os participantes foi distribuída folha com orientações gerais detalhando cuidados com os pés. Para os diagnosticados com NPD, também foi distribuída folha com orientações específicas para o acompanhamento dessa complicação.

\subsection{Potenciais vieses da aplicação do MNSI-BRASIL}

Um potencial viés apresentado pelo presente projeto piloto se refere à determinação da etiologia responsável pela polineuropatia, quando essa for reconhecida pelo instrumento MNSI. Várias entidades podem cursar com o mesmo acometimento no sistema nervoso periférico em situações clínicas específicas, a exemplo das causas alcóolicas, carenciais, infecciosas (ex: HIV), sarcoidoses, amiloidoses, vasculites ou mesmo paraneoplásicas; sendo que na maioria das vezes, as manifestações clínicas tampouco se diferem entre as diferentes etiologias (Evan et al., 2011).

A despeito disso, os inúmeros outros estudos que se propuseram a aplicar o instrumento MNSI não se valeram de critérios formais para separar eventuais etiologias diferenciais. Um estudo envolvendo 44 pacientes portadores de DM se propôs a comparar a efetividade do MNSI em relação ao teste isolado com monofilamentos, demonstrando significativa correlação entre esses métodos, mas sem esclarecer se houveram estratégias para descartar eventuais outras causas possíveis de neuropatias nessa amostra (De Souza Fortaleza et al., 2020).

Igualmente, outro trabalho conduziu a aplicação do MNSI em 1184 pacientes com DM, com objetivo de avaliar a performance desse instrumento na determinação de neuropatia, com vias a sugerir novos algoritmos ou possíveis adaptações em sua aplicação. Apesar de reconhecer a efetividade deste instrumento na deteçcão de neuropatia, especialmente com a utilização de um ponto de corte distinto do original, o estudo tem como âmago a proposta de detectar uma neuropatia nesse público, sem implicações etiológicas: sugere-se que DM seja uma importante causa desse distúrbio, mas não é possível confirmar se os indivíduos diagnosticados nesse estudo possuem de fato neuropatia diabética ou por alguma outra causa, já que não há critérios formais de exclusão para tais diagnósticos diferenciais (Herman et al., 2012).

Finalmente, tanto em sua criação quanto em sua adaptação transcultural, o MNSI projetou-se idealmente como um instrumento de determinação de neuropatia, o que lhe garante potencial promissor quando aplicado em populações sob risco aumentado de desenvolver essa condição, como a diabética. Conclui-se que sua aplicação como ferramenta de rastreio é fatidicamente útil para contextos clínicos específicos, já que pode suscitar uma investigação mais detalhada em muitos pacientes, com implicações terapêuticas. Contudo, em um cenário epidemiológico, faz-se necessário que estudos como este se valham de estratégias reais de exclusão dos demais diagnósticos diferenciais, de modo a obter uma estatística mais verossímil de uma neuropatia essencialmente diabética (Oliveira et al., 2016).

Revisando-se, então, os fatores que podem guiar essa diferenciação, destaca-se um trabalho envolvendo fatores de risco para neuropatia periférica em pacientes HIV+, que concluiu que os principais fatores determinantes para essa evolução são a longa duração de infecção por HIV e a contagem de CD4 <200 $\mu$ l (Evan et al., 2011).

Por outro lado, uma revisão acerca da polineuropatia alcóolica demonstra que para essa etiologia, as principais características sugestivas são uma natureza menos dolorosa, predominantemente sensitiva, em pacientes com alta dose de consumo alcoólico durante a vida. Outros fatores de risco incluem o padrão de consumo, a história de abuso de álcool na família, 
sexo masculino e mutações em ALDH2 (Julian et al., 2019). Um outro estudo revela que o consumo diário >100ml de álcool, especialmente em pacientes com doença hepática ou macrocitose, sugere a doença (Vittadini et al., 2001).

Tratando-se da etiologia carencial, um estudo de caso sobre um grupo de índios Xavantes portadores de polineuropatia concluiu que a deficiência de tiamina (vitamina B1) era responsável por esse padrão de acometimento neurológico, sabidamente associado a casos de desnutrição grave, erros alimentares, estados hipercatabólicos ou alcoolismo (Vieira Filho et al., 1997).

Outras causas mais raras podem ser sugeridas em contextos clínicos particulares, como as vasculites em caso de quadros agudos, assimétricos ou em pacientes sabidamente portadores dessas condições (Nichols \& Karivelil, 2020); ou as neoplasias, em pacientes com tumores diagnosticadas ou com síndromes paraneoplásicas típicas, incluindo prurido crônico associado a polineuropatia (Beigi et al., 2018); ou mesmo sarcoidose e amiloidose em pacientes sabidamente portadores dessas condições.

Cruzando-se todos esses fatores, sugere-se que o emprego conjunto de algumas dessas variáveis (contagem CD4, história de abuso de álcool, infecção pelo HIV, desnutrição ou diagnóstico prévio de doenças sistêmicas) durante a seleção amostral, poderia aumentar a probabilidade de que a amostra avaliada seja, unicamente, portadora de uma neuropatia diabética, superando-se o viés apresentado pelo presente estudo (De Souza Fortaleza et al., 2020; Vittadini et al., 2001; Nichols \& Karivelil, 2020; Beigi et al., 2018; Asad et al., 2010)

\subsection{Comparação entre escalas de avaliação de NPD}

Procedeu-se, também, breve revisão de literatura acerca de diversos instrumentos para rastreio e diagnóstico de NPD. Por fim, conforme disposto no Quadro 4, optou-se por comparar a MNSI-BRASIL com outros 3 instrumentos que possuem utilização recorrente na prática clínica e científica: Toronto Clinical Scoring System (TCNS), Neuropathy Disability Score (NDS) e Semmes-Weinstein Monofilaments Examination (SMWE).

Quadro 4 - Comparação entre instrumentos diagnósticos de NPD.

\begin{tabular}{|l|c|c|c|c|}
\hline \multicolumn{1}{|c|}{ Parâmetros avaliados } & MNSI-BRASIL & TCNS & NDS & SMWE \\
\hline Especificidade & $94 \%$ & $75,6 \%$ & $47.6 \%$ & $94 \%$ \\
\hline Sensibilidade & $79 \%$ & $77,2 \%$ & $92,3 \%$ & $82 \%$ \\
\hline Pesquisa de sintomas? & Sim & Sim & Não & Não \\
\hline Possui parte auto aplicada? & Sim & Não & Não & Não \\
\hline Possui exame físico? & Sim & Sim & Sim & Sim \\
\hline Teste de reflexo de tornozelo & Sim & Sim & Sim & Não \\
\hline Teste de reflexo de joelho & Não & Sim & Não & Não \\
\hline Diagnóstico a partir de pontuação & Sim & Sim & Sim & Não \\
\hline Teste de sensibilidade tátil & Sim & Sim & Sim & Sim \\
\hline Teste de sensibilidade térmica & Não & Sim & Sim & Não \\
\hline Teste de sensibilidade dolorosa & Não & Sim & Sim & Não \\
\hline Teste de sensibilidade propioceptiva & Sim & Sim & Sim & Não \\
\hline Teste de sensibilidade vibratória & Sim & Sim & Sim & Não \\
\hline
\end{tabular}

Fonte: Bril \& Perkins (2002); De Mettelinge et al. (2013); Freitas et al. (2009); Feng et al. (2009); Oliveira et al., (2016).

Os diversos tipos de sensibilidade são testados por inúmeros instrumentos. Assim, são testados reflexos do tornozelo, sensibilidade tátil, térmica e dolorosa, vibratória e proprioceptiva. Dentre essas, a sensibilidade tátil foi a única presente em todos os testes (De Mettelinge et al., 2013; Freitas et al., 2009; Feng et al., 2009).

Por outro lado, apenas a SWME não testa o reflexo do tornozelo, ao passo que a TCNS é a única que testa reflexo do joelho. Apenas a SMWE não gera diagnóstico a partir de pontuação. Quanto à sensibilidade térmica e dolorosa, apenas a NDS e TCNS avaliam esses parâmetros. As sensibilidades vibratória e proprioceptiva, por outro lado, são testadas por todas as escalas, exceto a SMWE (De Mettelinge et al., 2013; Freitas et al., 2009; Feng et al., 2009). 
Ao comparar-se as diversas escalas disponíveis para rastreio de neuropatia, observa-se que a MNSI-BRASIL apresenta especificidade próxima a 100\% para diagnostico de NPD, sendo esse nível muito semelhante à SMWE (Oliveira et al., 2016; Feng et al., 2009). Juntas, essas duas apresentam superioridade considerável se comparadas com a TCNS e NDS. Contudo, em relação à sensibilidade, o MNSI é inferior a todas as demais escalas.

Com relação aos instrumentos de pesquisa de neuropatia, apenas no MNSI-BRASIL e TCNS a história clínica do paciente possui relevância. Os demais, por sua vez, contam apenas com realização de exame físico (Bril \& Perkins, 2002; Feng et al., 2009).

\section{Conclusão}

Em linhas gerais, o presente trabalho demonstrou que o desenho de estudo empregado e o instrumento de avaliação são válidos e viáveis, muito embora algumas questões se colocaram como dificultadoras de sua conclusão prática, e são aqui descritas como potenciais vieses a serem contornados em casuísticas futuras.

No que tange ao desenho de estudo, a determinação da prevalência de NPD esbarrou em entraves já na seleção amostral, dadas as limitações e desatualizações do sistema de informação vigente, além de alguns fatores logísticos como o transporte dos participantes à ESF de referência. Contudo, observou-se que essas limitações são gargalos crônicos do sistema de saúde atual, e a sua resolução mostra-se imperiosa não só para a determinação da prevalência de NPD, mas para a qualidade do sistema assistencial como um todo.

Quanto ao instrumento empregado, o MNSI-Brasil mostrou-se uma ferramenta de rastreio útil a ser utilizada em contexto clínico específico. Porém, levanta-se como possíveis limitações a necessidade de alfabetização para a realização da parte auto aplicada, como também a incapacidade de exclusão dos diagnósticos diferenciais de NPD unicamente pelo instrumento. Contudo, considerando-se que não há um método padrão-ouro para determinação da NPD, e a partir da análise comparativa de outros instrumentos correlatos, ficou evidente que sua eficácia é pelo menos comparável ou superior em vários parâmetros de avaliação, o que endossa a sua escolha final.

Finalmente, ao analisar-se os resultados e discussões apresentados, o presente trabalho mostrou-se promissor ao apresentar uma perspectiva nova para a determinação da prevalência de NPD; e no intercurso desse processo vários ganhos secundários foram observados, como a discussão crítica a respeito dos instrumentos de saúde pública, oportunizando um momento único de retorno dos usuários ao sistema de saúde, com um estudo bibliográfico aprofundado e comparativo sobre os principais métodos diagnósticos para NPD, motivo pelo qual esse construto servirá de substrato para estudos futuros de prevalência de tal condição em adultos com DM 2.

Para tal, sugere-se que esses novos trabalhos que se proponham a avaliar a prevalência de NPD se valham de alguns ajustes metodológicos, a saber: inicialmente, recomenda-se buscar a planilha de usuários do serviço mais atualizada que esteja disponível, de modo a minimizar inconsistências temporais (como pacientes já falecidos ou não mais residentes do município). Adicionalmente, apesar do instrumento MNSI ter se mostrado efetivo para a avaliação da NPD, sugere-se complementá-lo com a coleta de alguns dados como a história de abuso de álcool, de infecção pelo HIV, de desnutrição ou de diagnóstico prévio de doenças sistêmicas (neoplasias, sarcoidoses, amiloidoses), haja visto que esses fatores foram altamente sugestivos de uma neuropatia de causa não-diabética, conforme mencionado anteriormente, de modo a se adicioná-los como critérios de exclusão para a amostragem futura.

Ademais, um cuidado adicional durante a impressão da escala em sua parte auto aplicada seria a utilização de uma fonte maior, para ao menos facilitar a leitura dos pacientes, possivelmente já portadores de restrições de acuidade visual secundárias ao próprio diabetes (retinopatia diabética). Recomenda-se, também, que sejam desenvolvidos novos estudos com a finalidade de 
se criar novos instrumentos, ou mesmo de se analisar a aplicabilidade e acurácia de testes já preexistentes para a avaliação da neuropatia periférica diabética. Desse modo, pode-se gerar testes de fácil aplicação, mas com significativa sensibilidade e especificidade, para que desta maneira seja possível realizar um diagnóstico e uma intervenção precoce sobre a condição estudada, de forma alinhada com os objetivos da prevenção terciária em saúde.

\section{Referências}

Asad, A., Hameed, M. A., Khan, U. A., Ahmed, N., \& Butt, M. U. R. A. (2010). Reliability of the neurological scores for assessment of sensorimotor neuropathy in type 2 diabetics. The Journal of the Pakistan Medical Association, 60(3), 166.

Beigi, M., Häberle, M., Gschwendtner, A., Baum, U., \& Weisshaar, E. (2018). Generalized chronic itch as a first sign of malignancy resembling paraneoplastic sensomotoric neuropathy. Acta dermato-venereologica, 98(5-6), 526-527.

Bittar, O. J. N., Biczyk, M., Serinolli, M. I., Novaretti, M. C. Z., \& de Moura, M. M. N. (2018). Sistemas de informação em saúde e sua complexidade. Revista de Administração em Saúde, 18(70).

Bril, V., \& Perkins, B. A. (2002). Validation of the Toronto Clinical Scoring System for diabetic polyneuropathy. Diabetes care, $25(11), 2048-2052$.

Brinati, L. M., Diogo, N. A. S., Moreira, T. R., Mendonça, É. T., \& Amaro, M. O. F. (2017). Prevalência e fatores associados à neuropatia periférica em indivíduos com diabetes mellitus Prevalence and factors associated with peripheral neuropathy in individuals with diabetes mellitus. Revista de Pesquisa Cuidado é Fundamental Online, 9(2), 347-355.

De Mettelinge, T. R., Delbaere, K., Calders, P., Gysel, T., Van Den Noortgate, N., \& Cambier, D. (2013). The impact of peripheral neuropathy and cognitive decrements on gait in older adults with type 2 diabetes mellitus. Archives of physical medicine and rehabilitation, 94(6), 1074-1079.

De Souza Fortaleza, A. C., Martinelli, A. R., Nozabieli, A. J. L., Mantovani, A. M., de Camargo, M. R., Fregonesi, C. E. P. T., \& de Faria, C. R. S. (2010). Avaliação clínica da sensibilidade em indivíduos com diabetes melito. In Colloquium Vitae, 2 (2), 44-49.

Evans, S. R., Ellis, R. J., Chen, H., Yeh, T. M., Lee, A. J., Schifitto, G., Wu, K., Bosch, R. J., McArthur, J. C., Simpson, D. M., \& Clifford, D. B. (2011). Peripheral neuropathy in HIV: prevalence and risk factors. AIDS (London, England), 25(7), 919-928.

Feng, Y., Schlösser, F. J., \& Sumpio, B. E. (2009). The Semmes Weinstein monofilament examination as a screening tool for diabetic peripheral neuropathy. Journal of vascular surgery, 50(3), 675-682.

Freitas, C., Carvalho, A., Melo-Rocha, G., Amaral, C., Pinto, S., \& Guimarães, R. (2009). O teste com Neuropad na detecção precoce da neuropatia periférica do doente diabético. Acta MedPort [Internet], 22(6), 729-734.

Fundação João Pinheiro. Governo de Minas Gerais. (2018). Estatísticas da Educação em Minas Gerais. http://fjpdados.fjp.mg.gov.br/Educacao/\#: :text=Os\%20dados\%20da\%20Pesquisa,cerca\%20de\%201\%2C03\%20mil

Herman, W. H., Pop-Busui, R., Braffett, B. H., Martin, C. L., Cleary, P. A., Albers, J. W., Feldman, E. L., \& DCCT/EDIC Research Group (2012). Use of the Michigan Neuropathy Screening Instrument as a measure of distal symmetrical peripheral neuropathy in Type 1 diabetes: results from the Diabetes Control and Complications Trial/Epidemiology of Diabetes Interventions and Complications. Diabetic medicine : a journal of the British Diabetic Association, 29(7), 937944.

Intitituto Brasileiro de Geografia e estatística. (2020). Agência de notícias IBGE. PNAD Educação 2019: Mais da metade das pessoas de 25 anos ou mais não completaram o ensino médio. https://agenciadenoticias.ibge.gov.br/agencia-sala-de-imprensa/2013-agencia-de-noticias/releases/28285-pnad-educacao-2019mais-da-metade-das-pessoas-de-25-anos-ou-mais-nao-completaram-o-ensino-medio

Julian, T., Glascow, N., Syeed, R., \& Zis, P. (2019). Alcohol-related peripheral neuropathy: a systematic review and meta-analysis. Journal of neurology, 266(12), 2907-2919.

Koche, J. C. (2011). Fundamentos de Metodologia Científica: teoria da ciência e iniciação à pesquisa. Petrópolis: Vozes.

Li, J., Huang, D. Q., Zou, B., Yang, H., Hui, W. Z., Rui, F., Yee, N., Liu, C., Nerurkar, S. N., Kai, J., Teng, M., Li, X., Zeng, H., Borghi, J. A., Henry, L., Cheung, R., \& Nguyen, M. H. (2021). Epidemiology of COVID-19: A systematic review and meta-analysis of clinical characteristics, risk factors, and outcomes. Journal of medical virology, 93(3), 1449-1458.

Lima, S. A. V., Silva, M. R. F. D., Carvalho, E. M. F. D., Pessoa, E. Â. C., Brito, E. S. V. D., \& Braga, J. P. R. (2015). Elementos que influenciam o acesso à atenção primária na perspectiva dos profissionais e dos usuários de uma rede de serviços de saúde do Recife. Physis: Revista de Saúde Coletiva, $25,635-656$.

Medronho, R. A., Bloch, K. V., Luiz, R. R., \& Werneck, G. L. (2009). Epidemiologia. (2a ed): Atheneu.

Moreira, R. O., Castro, A. P., Papelbaum, M., Appolinário, J. C., Ellinger, V., Coutinho, W. F., \& Zagury, L. (2005). Tradução para o português e avaliação da confiabilidade de uma escala para diagnóstico da polineuropatia distal diabética. Arquivos Brasileiros de Endocrinologia \& Metabologia, $49,944-950$.

Nascimento, O. J. M. D., Pupe, C. C. B., \& Cavalcanti, E. B. U. (2016). Diabetic neuropathy. Revista Dor, $17,46-51$. 
Research, Society and Development, v. 10, n. 11, e221101119568, 2021 (CC BY 4.0) | ISSN 2525-3409 | DOI: http://dx.doi.org/10.33448/rsd-v10i11.19568

Nichols, L., \& Karivelil, M. J. (2020). Vasculitis with a neuromuscular presentation and associated cancer revealed by autopsy. Autopsy and Case Reports, 10.

Ochoa-Vigo, K., Torquato, M. T. D. C. G., Silvério, I. A. D. S., Queiroz, F. A. D., \& Pace, A. E. (2006). Caracterização de pessoas com diabetes em unidades de atenção primária e secundária em relação a fatores desencadeantes do pé diabético. Acta Paulista de Enfermagem, 19(3), $296-303$.

Oliveira, F. B. D., Botelho, K. K. P., Bezerra, A. R., Azevedo, D. I. D. O., Santos-Couto-Paz, C. C. D., \& Fachin-Martins, E. (2016). Cross-cultural adaptation to Brazilian Portuguese of the Michigan neuropathy screening instrument: MNSI-Brazil. Arquivos de neuro-psiquiatria, 74, 653-661.

Oliveira, F. B. (2015) Opções diagnósticas no monitoramento de neuropatias diabéticas: em busca de parâmetros para tomada de decisões clínicas. [Tese doutorado, Universidade de Brasília]. https://repositorio.unb.br/handle/10482/19410

Reis, E., Melo, P., Andrade, R., \& Calapez, T. (2006). Estatística aplicada. (4a ed.): Edições Sílabo.

Sociedade Brasileira de Diabetes (2019). Diretrizes da Sociedade Brasileira de Diabetes 2019-2020.

Schmidt, M. I., Duncan, B. B., Azevedo e Silva, G., Menezes, A. M., Monteiro, C. A., Barreto, S. M., Chor, D., \& Menezes, P. R. (2011). Chronic noncommunicable diseases in Brazil: burden and current challenges. Lancet, 377(9781), 1949-1961.

Schramm, J. M. D. A., Oliveira, A. F. D., Leite, I. D. C., Valente, J. G., Gadelha, Â. M. J., Portela, M. C., \& Campos, M. R. (2004). Transição epidemiológica e o estudo de carga de doença no Brasil. Ciência \& Saúde Coletiva, 9, 897-908

Tesser, C. D., Norman, A. H., \& Vidal, T. B. (2018). Acesso ao cuidado na Atenção Primária à Saúde brasileira: situação, problemas e estratégias de superação. Saúde em Debate, 42, 361-378.

Vieira Filho, J. P. B., Oliveira, A. S. B., Da Silva, M. R. D., Amaral, A. L., \& Schultz, R. R. (1997). Polineuropatia nutricional entre índios Xavantes. Revista da Associação Médica Brasileira, 43, 82-88.

Vilar, L. (2016). Endocrinologia Clínica. (6a ed.): Guanabara Koogan.

Vittadini, G., Buonocore, M., Colli, G., Terzi, M., Fonte, R., \& Biscaldi, G. (2001). Alcoholic polyneuropathy: a clinical and epidemiological study. Alcohol and alcoholism. 36(5), 393-400. 